\title{
Cell Proliferation Study of Human Osteosarcoma Cell Line (U2OS) using Alamar Blue Assayand Live Cell Imaging
}

\author{
Marahaini Musa ${ }^{1}$, Thirumulu Ponnuraj Kannan ${ }^{1,2, *}$, Shyamoli Mustafa ${ }^{3}$ \\ ${ }^{1}$ School of Dental Sciences, UniversitiSains Malaysia, 16150 KubangKerian, Kelantan, Malaysia \\ ${ }^{2}$ Human Genome Centre, School of Medical Sciences, UniversitiSains Malaysia, 16150 KubangKerian, \\ Kelantan, Malaysia \\ ${ }^{3}$ School of Health Sciences, UniversitiSains Malaysia, 16150 KubangKerian, Kelantan, Malaysia
}

\begin{abstract}
Cell proliferation can be described as the process in which cells reproduce themselves by growing and then dividing into two equal copies. The cancer cell differs from the normal cell in a sense that it is aberrantly regulated. Failure to regulate functions of biomolecules that are necessary for survival, proliferation, differentiation as well as expression of many cell-type functions leads to an altered phenotype and cancer. The aim of the study is to assess the proliferation of human osteosarcoma cell line (U2OS) using Alamar Blue and live cell imaging.Standard curve for U2OS cells was produced by incubating different counts of U2OS cells for 24 hours before addition of Alamar Blue dye and absorbance reading at $570 \mathrm{~nm}$ and $600 \mathrm{~nm}$. For cell proliferation study, $1 \times 10^{3}$ of U2OS cells were cultured for 10 days. Absorbance readings were recorded once in every two days (day 2, 4, 6, 8, 10) and the percentage of Alamar Blue reduction was calculated. The cell division process was studied using live cell imaging for 48 hours employing fluorescent expression systems. From both Alamar Blue assay and live cell imaging, population doubling time (PDT) was determined. The percentage of Alamar Blue reduction increased from day 2 to 10 which indicated the increase in the number and proliferation activity of U2OS cells. The PDT of U2OS cells determined based on Alamar Blue assay was 29.15 hours and through live cell imaging, was $32 \pm 3$ hours.U2OS cells proliferate over days based on Alamar Blue assay and live cell imaging with PDT of approximately 29 hours.
\end{abstract}

Keywords - osteosarcoma, cell proliferation, Alamar Blue assay, live cell imaging, population doubling time

Accepted Date: 25 .June 2013

\section{Introduction}

Cancer refers to over 200 diseases that are caused by cells proliferating in an out-of-control manner. The cancer cell differs from the normal cell in a sense that it is aberrantly regulated. The process of replication and division of cells are normally very strictly regulated, and cells are either repaired or undergo programmed cell death (apoptosis) as they become old, damaged or mutated [1]. Failure to regulate functions of biomolecules that are necessary for survival, proliferation, differentiation as well as expression of many cell-type functions leads to an altered phenotype and cancer[2].

Cell proliferation can be described as the process in which cells reproduce themselves by growing and then dividing into two equal copies [3]. The rate of proliferation of cells within any population of cells depends on several parameters/ factors such as $(a)$ the rate of division of cells $(\mathrm{Tc}),(b)$ the fraction of cells within the population undergoing cell division (growth fraction), and $(c)$ the rate of cell loss from the population due to terminal differentiation or cell death. Tc represents the time needed for a cell cycle to be completed. The cell cycle can be classified into two functional phases, $\mathrm{S}$ and $\mathrm{M}$ phases, and two preparatory phases, G1 and G2 [2].

$\mathrm{S}$ phase of cell cycle is known as DNA synthesis phase, and segregation of complete sets of chromosomes to each of the daughter cells occurs in M phase. "Gap" phases includes G1, which connects the completion of $M$ phase to initiation of $S$ phase in the next cycle, and $G 2$, which separates the $S$ and $M$ phases. Depending on the environmental and developmental signals, cells in G1maytemporarily or permanently leave the cell cycle and enter G0 (a quiescent or arrested phase) [4].

As cancer cells produce different rate of proliferation compared to normal cells, the aim of this study was to assess the proliferation of human osteosarcoma cell line (U2OS) using Alamar Blue and live cell imaging as well as to determine the time duration for U2OS cells to double and produce two daughter cells.

\subsection{Cell culture}

\section{Methods}

Human osteosarcoma cell line, U2OS (ATCC Catalog No. HTB-96, USA) was cultured in RPMI 1640 culture medium (Gibco, USA) supplemented with L-Glutamine (Gibco, USA), $10 \%$ fetal bovine serum 
(GibcoUSA) and $1 \%$ of $5000 \mathrm{IU}$ of penicillin and $5000 \mu \mathrm{g}$ of streptomycin per $\mathrm{ml}$ (Gibco, USA) in humidified incubator at $37^{\circ} \mathrm{C}$ and $5 \% \mathrm{CO}_{2}$ for 3 days until it reached confluence. Then, the cells were washed with phosphate buffered saline (Gibco, USA) and trypsinized using $0.25 \%$ trypsin-EDTA solution (Gibco, USA). The cells were then centrifuged and the cell pellet was re-suspended with culture medium before the cells were seeded for the test.

\subsection{Alamar Blue assay}

Standard curve for U2OS cells was produced by incubating different counts of cells $(391,781,1563$, $3125,6250,12500,25000,50000$ ) for 24 hours before addition of Alamar Blue dye (Invitrogen, UK) and absorbance reading at $570 \mathrm{~nm}$ and $600 \mathrm{~nm}$. Percentage of reduction was counted using the formula below [5]: $\%$ reduction $=[(117.216) \mathrm{A} 570-(80.586) \mathrm{A} 600] /\left[(155.677) \mathrm{A}^{\prime} 600-(14.652) \mathrm{A}^{\prime} 570\right] \times 100 \%$ Where 117.216 is the molar extinction coefficient of Alamar Bluein the oxidized form at $600 \mathrm{~nm}$; 80.586 is the molar extinction coefficient of Alamar Bluein the oxidized form at $570 \mathrm{~nm} ; 14.652$ is the molar extinction coefficient of Alamar Bluein the reduced form at $600 \mathrm{~nm} ; 155.677$ is the molar extinction coefficient of Alamar Bluein the reduced form at $570 \mathrm{~nm} ; \mathrm{A}_{600}$ is the absorbance of test wells at $600 \mathrm{~nm} ; \mathrm{A}_{570}$ is the absorbance of test wells at $570 \mathrm{~nm} ; \mathrm{A}_{600}^{\prime}$ is the absorbance of negative control wells at $600 \mathrm{~nm} ; \mathrm{A}_{570}^{\prime}$ is the absorbance of negative control wells at $570 \mathrm{~nm}$.

Standard curve for the U2OS cell line was constructed. For cell proliferation study, $1 \times 10^{3}$ of U2OS cells were seeded in 96-well plate and cultured for 10 days. Absorbance readings at $570 \mathrm{~nm}$ and $600 \mathrm{~nm}$ were recorded once in every two days (day 2, 4, 6, 8, 10) after the addition of Alamar Blue dye in each well and incubation for 4 hours. The test was performed in triplicates. The percentage of Alamar Blue reduction was counted and graph of percentage of reduction of the tested group over time was constructed. Higher value of percentage of reduction represented greater level of cell growth. Based on the standard curve and the graph of percentage of reduction, population doubling time (PDT) of U2OS cells was determined using the following formula [6]:

$$
\mathrm{PDT}=1 / \mathrm{r}
$$

Where, multiplication rate $(r)=3.32\left(\log \mathrm{NH}-\log \mathrm{N}_{1}\right) /\left(t_{2}-t_{1}\right)$; N1: cell number at day 2 (48 hours); NH: cell number at day 10 (240 hours); $t_{2}$ : end of experiment ( 240 hours); $t_{1}$ : start of the experiment (48 hours)

\subsection{Live cell imaging}

$1 \times 10^{4}$ of U2OS cells were seeded in $35 \mathrm{~mm}$ culture dish and $2 \mu \mathrm{l}$ of CellLight ${ }^{\mathrm{TM}}$ Histone-GFP (BacMam 2.0) and CellLight ${ }^{\mathrm{TM}}$ Tubulin-GFP (BacMam 2.0) fluorescent expression systems (Invitrogen, UK) were added to the cells. The cell division process was studied using live cell imaging for 48 hours whereby the duration of time for a cell to divide (PDT) was noted.

\section{Results}

The percentage of Alamar Blue reduction increased proportionally with cell count whereby 50000 U2OS cells produced the highest percentage of reduction compared to lower cell count. From the constructed standard reference curve, the percentage of Alamar Blue reduction increased tremendously from cell count of 0 to 10000 cells (Fig. 1).

In Alamar Blue assay, the percentage of reduction of U2OS cells also increased from day 2 to 10 . This indicated the increase in the number and proliferation activity of the cells (Fig. 2). This was evidently supported by the calculated U2OS cell number based on the results of Alamar Blue assay (Table 1). The PDT of U2OS cells determined based on Alamar Blue assay was 29.15 hours.

In live cell imaging, the morphology of U2OS cell was observed starting at 0 hour and ended at 48 hours (Fig. 3). The cell appeared elongated in shape and as the time proceeded the cells became roundedprior to cell division. The nuclear division could be clearly seen due to the green fluorescent expression of the histone and tubulin. Cytokinesis followed shortly after that and the two daughter cells then were separated from each other. These cells then also divided after several hours in incubation. The process of division of cells is shown in Fig. 4. Through live cell imaging, the PDT of U2OS cell was determined as $32 \pm 3$ hours.

\section{Discussion}

U2OS cells are osteosarcoma cells, which are derived from malignant bone tumors. These cancer cells share some osteoblastic features [7], but the alteration in the chromosomes lead to abnormal molecular and cellular functions [8]. Proliferation of U2OS cells was studied in the present study using Alamar Blue assay. This assay got its name from the Alamar Blue (AB), which is a tetrazolium-based dye, incorporating resazurin and resorufin as oxidation-reduction indicators that produce colorimetric changes and a fluorescent signal in response to metabolic activity where the blue non-fluorescent oxidised form turns to pink and fluorescent upon reduction $[9,10]$. For many decades, the Alamar Blue assay has been applied as a quantitative analysis of 
viability and/ or proliferation of many organisms including several normal and cancerous cell lines [11]. The advantages of Alamar Blue assay are accurate time-course measurements, possess high sensitivity and linearity, involves no cell lysis, suitable for use with post-measurement functional assays, can be used with different cell models (flexibility), is scalable and compatible with fluorescence- and/or absorbance-based instrumentation platforms, and importantly, non-toxic, non-radioactive and is safe for the user, and the environment [12].

The three major parameters that are applied to characterize cell growth are specific growth rate $(\mu)$, population doubling time (PDT), lag time and maximum cell density [13]. Previous study on the proliferation of several osteosarcoma cell lines which includes U2OS had reported that the number of osteosarcoma cell lines increased with duration of incubation and reached the saturation density at approximately $10,000 \mathrm{cells} / \mathrm{cm}^{2}$ in which the cells goes into plateau (stationary) growth phase at density higher than stated and finally entered the decline growth phase [14]. This finding supported the result of standard curve of U2OS cells in which exponential growth were seen at cell count lower than 10000 cells. However, the growth rate became less and becoming more plateau as the cell number increased. This may be due to depletion of the essential elements of growth environment which includes dissolved oxygen [DO] concentration, nutrient concentrations, temperature, $\mathrm{pH}$, etc [13]. In the Alamar Blue assay which was conducted for 10 days, the U2OS cell number also increased with time and the curve of the cells (Fig. 2) indicated the log phase whereby the viable cell count increased exponentially.

In the present study, live cell imaging was performed by employing fluorescent expression system in which two specific organelles/ cellular structures were targeted: tubulin and histone. The physical process of duplicated chromosomes segregation happens on one of the most striking and dynamic of all sub-cellular structures-the mitotic spindle. The mitotic spindle consists of microtubules which extend from each spindle pole and connect to the kinetochore region of chromosomes. Microtubules are composed of two tubulin subunits $(\alpha$ and $\beta$ tubulin), and lengthening and shortening of microtubule are important to segregate chromosomal DNA [15]. Histones are highly alkaline proteins found in eukaryotic cell nuclei that package and order the DNA into structural units known as nucleosomes [16, 17]. It also constitutes half of the mass of chromatin [18]. Due to these special characteristics of histone and tubulin, they were chosen as the cellular structure of interest to be observed.

The determination of PDT was performed by the calculation based on the results of Alamar Blue assay. As this was the quantitative measure for the PDT determination, the qualitative determination of PDT was done by observing the cell division using live cell imaging. Even though the number of U2OS cells can be clearly seen in the microscope field in live cell imaging system and the comparison of the cell number between the start and the end of the experiment can be performed, the quantitative measure of PDT cannot be done as this will lead to possible error. Different cells possess different time to divide and the some cells may take longer or shorter time for cell division to be completed. For the determination of PDT using live cell imaging, only specific cells were focussed in each experiment in which they have undergone two cell divisions (producing 4 daughter cells) throughout the duration of the test. This has to be done as only one cell division may not be sufficient for the exact determination of the duration for a cell to divide.

The stages of cell cycle can be determined partly from the morphology of the cells as the mitosis stage involves the separation of a cell into two identical copies. This process only takes several hours $(4 \pm 1$ hour $)$ which was a portion of the PDT ( $32 \pm 3$ hours). The remainder time suggested the occurrence of other phases of cell cycle (S, G1 and G2 phases). However, the exact determination of each stage of cell cycle could be done as no specific indicator for the cell cycle phases was used in this experiment.

The PDT produced by Alamar Blue assay ( 29.15 hours) and live cell imaging ( $32 \pm 3$ hours) differed even though the numbers were quite close. Considering the results of these tests, the approximate time for U2OS cell to divide is 29 hours. An exact time for a cell division cannot be done as individual cell hasa different time to be divided. This was supported by the one of the suppliers for U2OS cells which suggested the doubling time of U2OS cells to be approximately 29 hours [19]. A slight difference in the PDT could also result from the use of different culture medium where the composition in the medium plays a role in the growth of cell [13].

\section{Conclusion}

U2OS cells proliferate over days based on Alamar Blue assay and live cell imaging. The PDT of this specific cell line was approximately at 29 hours.

\section{Acknowledgements}

The authors would like to acknowledge the staff of Craniofacial Sciences Laboratory, School of Dental Sciences and USM incentive grant 2012/0971 for the financial support. 


\section{References}

[1] B. Denard, C. Lee, and J. Ye, Doxorubicin blocks proliferation of cancer cells through proteolytic activation of CREB3L1, eLife, 1, 2012, e00090.

[2] M. Andreeff, D. Goodrich, and A. Pardee, in R. J. Bast, D. Kufe, R. Pollock, R.R. Weichselbaum, J. F. Holland, and E. Frei (Eds.), Holland-Frei Cancer Medicine, Fifth edition, chapter 2 (Hamilton, ON: BC Decker, 2000) Available from: http://www.ncbi.nlm.nih.gov/books/NBK20860/.

[3] M.J. Berridge, Cell Signalling Biology, 2012, doi: 10.1042/csb0001001.

[4] G. Cooper, The Cell: A Molecular Approach,Second edition(Sunderland, MA: Sinauer Associates, 2000) Available from: http://www.ncbi.nlm.nih.gov/books/NBK9876/.

[5] H.H. Willard, L.L. Merritt, and J. Dean, Instrumental Methods of Analysis,seventh edition(California, US: Wadsworth Publishing, 1965).

[6] J.P. Mather, and P.E.Roberts, Introduction to Cell and Tissue Culture: Theory and Technique,(New York, US: Plenum Press, 1998)

[7] H. Declercq, N. Van den Vreken, E. De Maeyer, R. Verbeeck, E. Schacht, L. De Ridder, and M. Cornelissen, Isolation, proliferation and differentiation of osteoblastic cells to study cell/biomaterial interactions: comparison of different isolation techniques and source. Biomaterials, 25 (5), 2004, 757-768.

[8] K. Al-Romaih, J. Bayani, J. Vorobyova, J. Karaskova, P.C. Park, M. Zielenska, and J.A. Squire, Chromosomal instability in osteosarcoma and its association with centrosome abnormalities. Cancer Genetics and Cytogenetics, 144 (2), $2003,91-99$.

[9] R.M. DeBaun, and G. de Stevens, On the mechanism of enzyme action. XLIV. Codetermination of resazurin and resorufin in enzymatic dehydrogenation experiments, Archives of Biochemistry and Biophysics,31 (2), 1951, 300-308.

[10] B. Page, M. Page, and C. Noel, A new fluorometric assay for cytotoxicity measurements in vitro, International Journal of Oncology, 30, 1993, 473-476.

[11] G.R. Nakayama, M.C. Caton, M.P. Nova, and Z. Parandoosh, Assessment of the Alamar Blue assay for cellular growth and viability in vitro. Journal of Immunological Methods, 204 (2), 1997, 205-208.

[12] S.N. Rampersad, Multiple applications of Alamar Blue as an indicator of metabolic function and cellular health in cell viability bioassays, Sensors, 12 (9),2012, 12347-12360.

[13] D.W. Murhammer, Useful Tips, Widely Used Techniques, and Quantifying Cell Metabolic Behavior, in D.W. Murhammer (Ed), Baculovirus and Insect Cell Expression Protocols, 388 (New York, US: Humana Press, 2007) 3-22.

[14] C. Pautke, M. Schieker, T. Tischer, A. Kolk, P. Neth, W. Mutschler, and S. Milz, Characterization of osteosarcoma cell lines MG63, Saos-2 and U-2 OS in comparison to human osteoblasts, Anticancer Research, 24 (6),2004, 3743-3748.

[15] C.P.C. De Souza, and S.A. Osmani, Mitosis, Not just open or closed, Eukaryotic Cell,6 (9),2007, $1521-1527$.

[16] R.M. Youngson, Collins Dictionary of Human Biology, (Glasgow, UK: HarperCollins, 2006).

[17] D.L. Nelson and M.M. Cox, Lehninger Principles of Biochemistry, Fourth edition(San Francisco, US: W.H. Freeman \& Co. Ltd, 2004).

[18] M.E. Ewen, Where the cell cycle and histones meet, Genes \& Developments, 14 (18), 2000, 2265-2270.

[19] Sigma-Aldrich. U2OS Cells GFP-NUP98. (Accessed on $1^{\text {st }}$ April 2013), Available from: http://www.sigmaaldrich.com/catalog/product/sigma/cll1136?lang=en\&region=US.

\section{Figures}

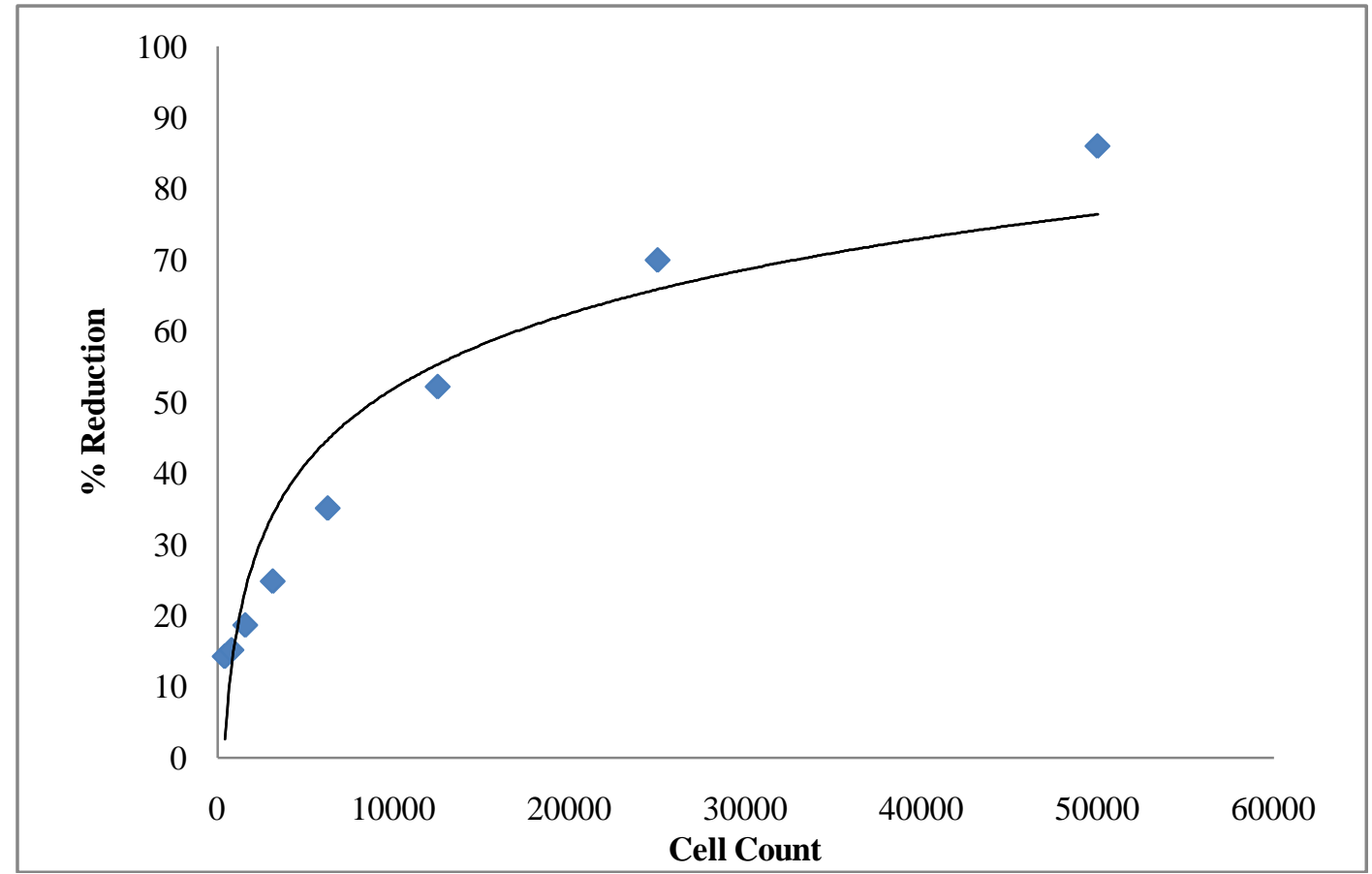

Figure 1: Standard curve of U2OS cells 


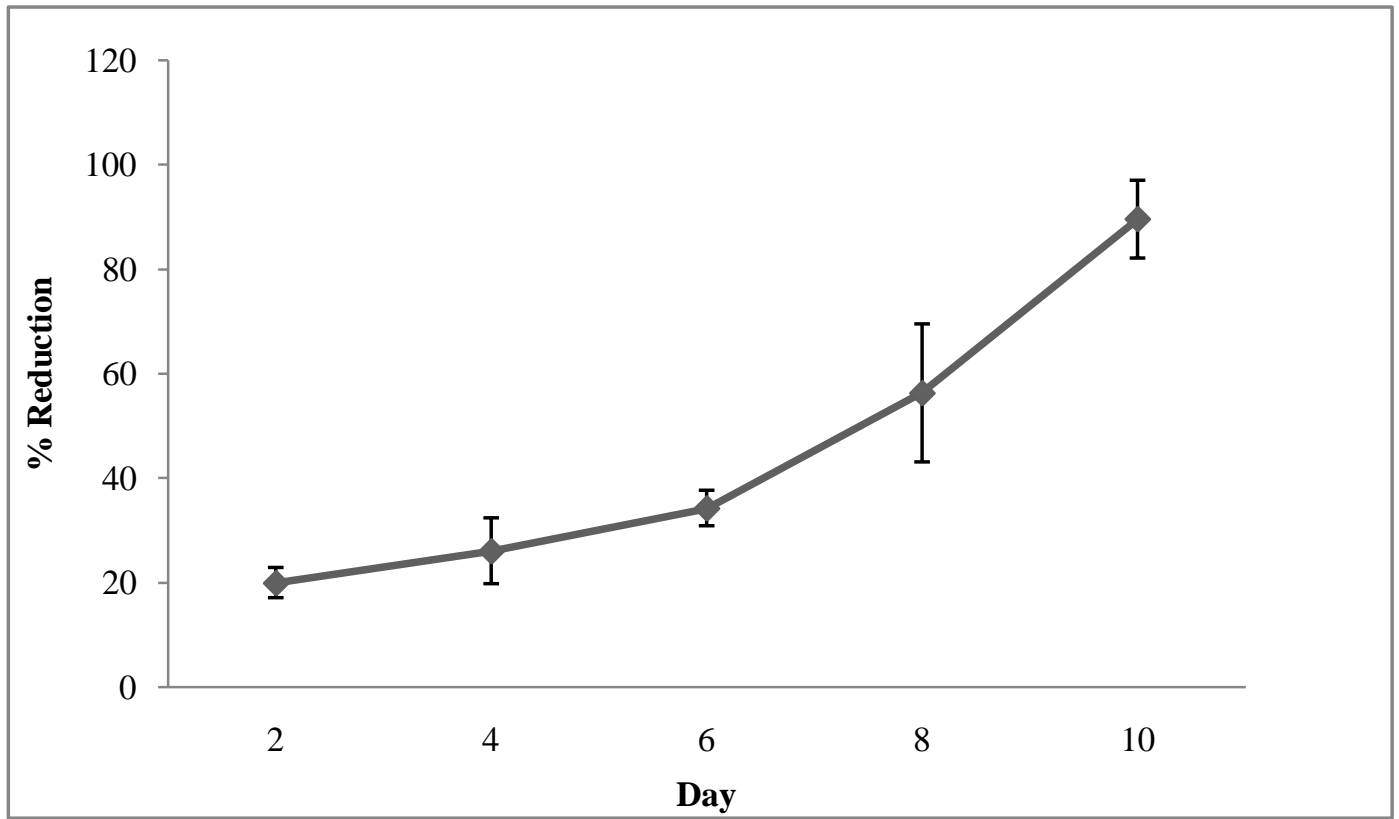

Figure 2: Percentage of reduction of U2OS cells from day 2 to day 10

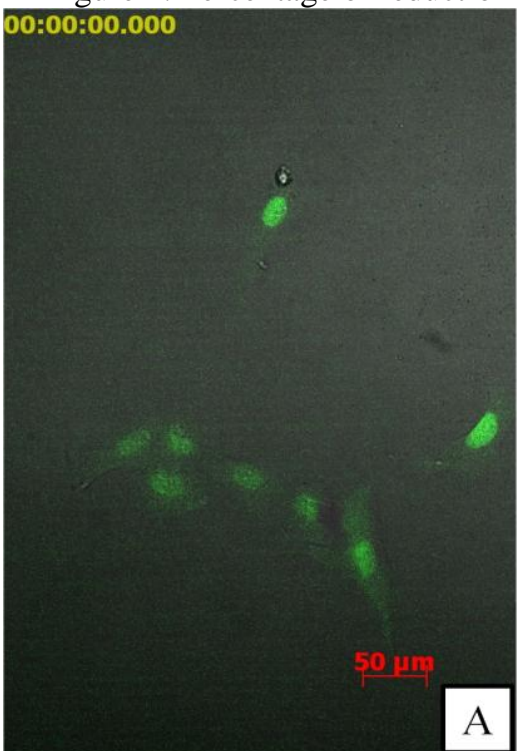

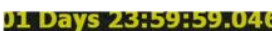

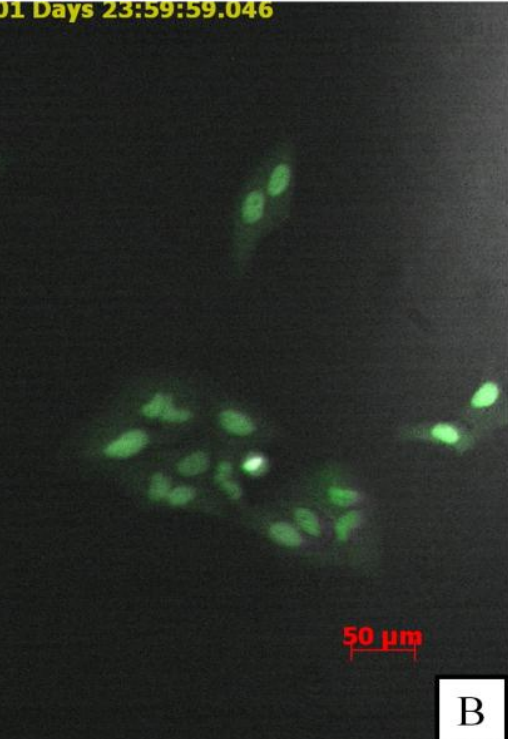

Figure 3: U2OS cell morphology based on live cell imaging A) at the start of experiment (0 hour); B) at the end of experiment (48 hours) 


\section{Cell Proliferation Study of Human Osteosarcoma Cell Line (U2OS) using Alamar Blue Assay and}
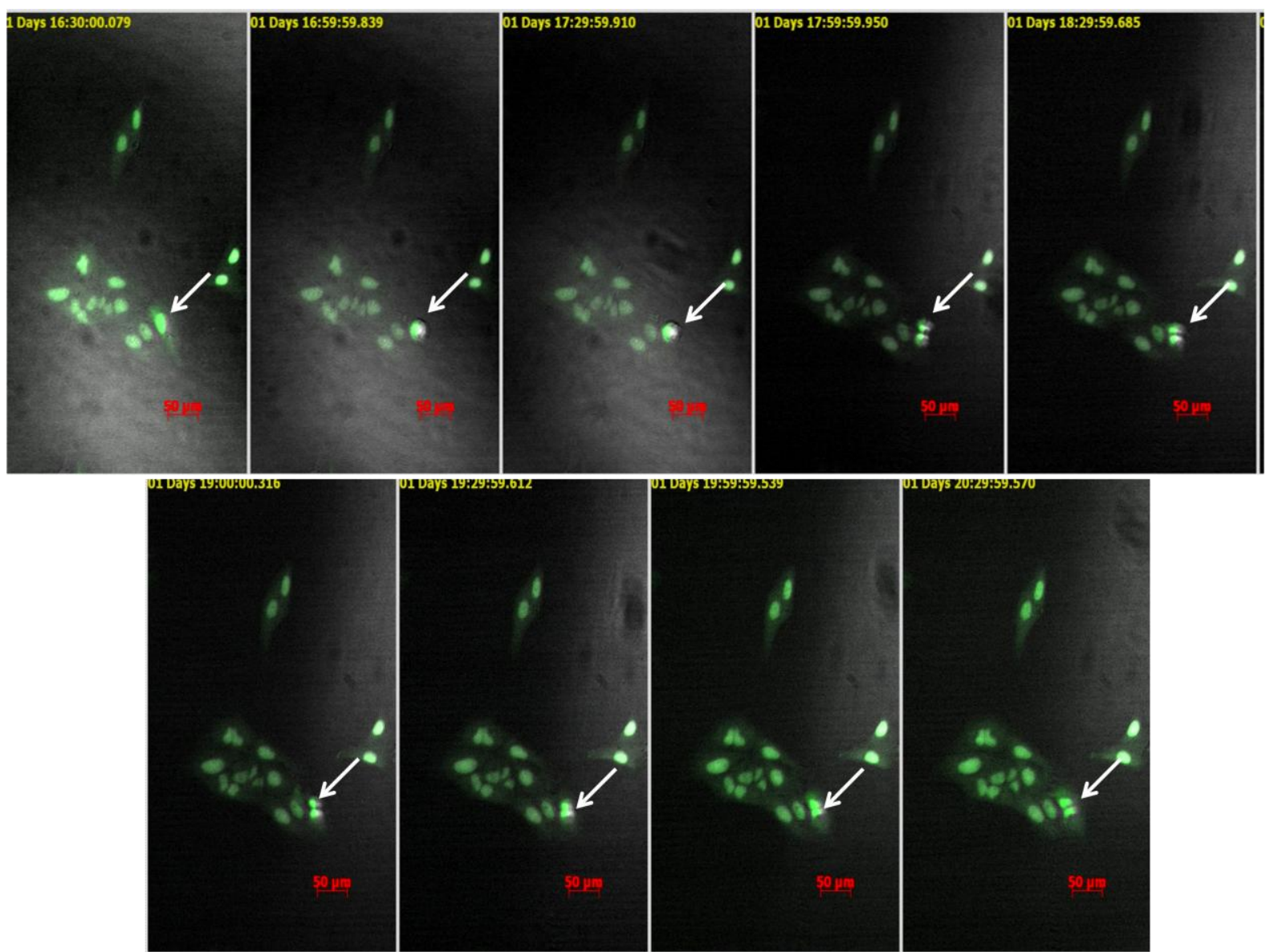

Figure 4: Cell division process of U2OS cells

Table

Table 1: The U2OS cell count in Alamar Blue assay

\begin{tabular}{ll}
\hline Day & Cell count \\
\hline 2 & 1228 \\
4 & 1927 \\
6 & 3128 \\
8 & 13279 \\
10 & 117824 \\
\hline
\end{tabular}

